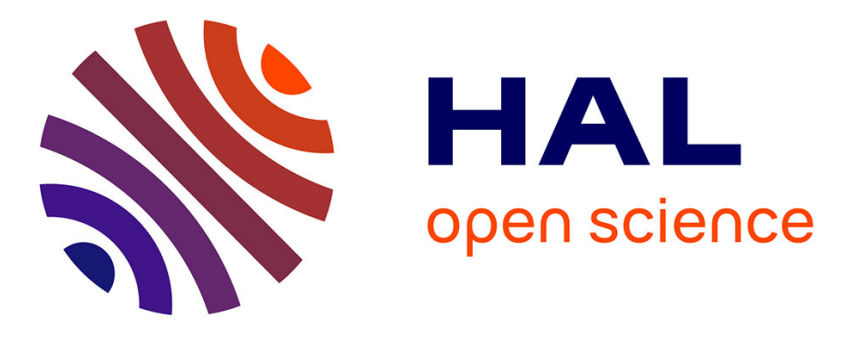

\title{
La campagne IHOP 2002 - Une campagne de mesure de la vapeur d'eau dans la couche limite
}

\author{
Cyrille Flamant, Françoise Guichard, Joël van Baelen, Olivier Bock, Fleur \\ Couvreux, David Parsons, Tammy Weckwerth, Jacques Pelon, Philippe \\ Drobinski, Karen Lhomme, et al.
}

\section{To cite this version:}

Cyrille Flamant, Françoise Guichard, Joël van Baelen, Olivier Bock, Fleur Couvreux, et al.. La campagne IHOP 2002 - Une campagne de mesure de la vapeur d'eau dans la couche limite. La Météorologie, 2003, 43, pp.38-47. 10.4267/2042/35999 . hal-02093057

\section{HAL Id: hal-02093057 https://hal.uca.fr/hal-02093057}

Submitted on 27 Jan 2020

HAL is a multi-disciplinary open access archive for the deposit and dissemination of scientific research documents, whether they are published or not. The documents may come from teaching and research institutions in France or abroad, or from public or private research centers.
L'archive ouverte pluridisciplinaire HAL, est destinée au dépôt et à la diffusion de documents scientifiques de niveau recherche, publiés ou non, émanant des établissements d'enseignement et de recherche français ou étrangers, des laboratoires publics ou privés. 


\title{
La campagne IHOP 2002
}

\author{
Une campagne de mesure \\ de la vapeur d'eau dans la couche limite
}

\section{Résumé}

Le projet IHOP 2002 (International H2O Project) s'est déroulé du 13 mai au 25 juin 2002 au-dessus des Grandes Plaines de l'Oklahoma. Il résulte d'une initiative des communautés scientifiques américaine et européenne. L'objectif scientifique principal est d'améliorer la caractérisation spatio-temporelle de la distribution de la vapeur d'eau dans l'atmosphère afin de mieux comprendre et prédire les phénomènes convectifs. La région présente l'avantage de posséder un réseau d'instruments (expérimentaux et opérationnels) déjà en place et de se situer dans une zone fréquemment caractérisée par de forts gradients d'humidité et très active du point de vue de la convection. Cet article expose les moyens mis en œuvre et la stratégie expérimentale. Une attention particulière est portée à la contribution de la communauté française. Enfin, quelques résultats préliminaires sont présentés.

\section{Abstract}

The IHOP 2002 experiment:

a field experiment on water vapor in the boundary layer

The International H2O Project (IHOP 2002) was a joint American and European field experiment that took place over the Southern Great Plains of the USA (Oklahoma) from 13 May to 25 June 2002. Its chief aim was to improve characterization of the four-dimensional distribution of water vapor and its application to improving the understanding and prediction of convection. The region has the advantage of existing experimental and operational facilities, strong variability in moisture, and active convection. In this paper, we present the means used as well as the experimental strategy. Particular attention is paid to the contribution of French scientists. Preliminary results are also presented.

\section{Cyrille Flamant $^{(1)}$, Françoise Guichard( ${ }^{(2)}$, Joël van Baelen ${ }^{(2)}$, Olivier Bock $^{(1)}$, Fleur Couvreux ${ }^{(2)}$, David Parsons ${ }^{(3)}$, Tammy Weckwerth ${ }^{(3)}$, Jacques Pelon ${ }^{(1)}$, Philippe Drobinski ${ }^{(1)}$, Karen Lhomme ${ }^{(1)}$, Mikael Guenno $^{(2)}$ et Erik Doerflinger ${ }^{(4)}$}

(1) Institut Pierre-Simon Laplace - Service d'aéronomie, CNRS Université Pierre et Marie Curie - Boîte 102, 5 place Jussieu, 75252 Paris Cedex 05 cyrille.flamant@aero.jussieu.fr

(2) Météo-France Centre national de recherches météorologiques (CNRM) - Toulouse

(3) National Center for Atmospheric Research - Atmospheric Technology Division Boulder Co, ÉTATS-UNIS

(4) Laboratoire de la dynamique de la lithosphère CNRS et université Montpellier-2 - Montpellier

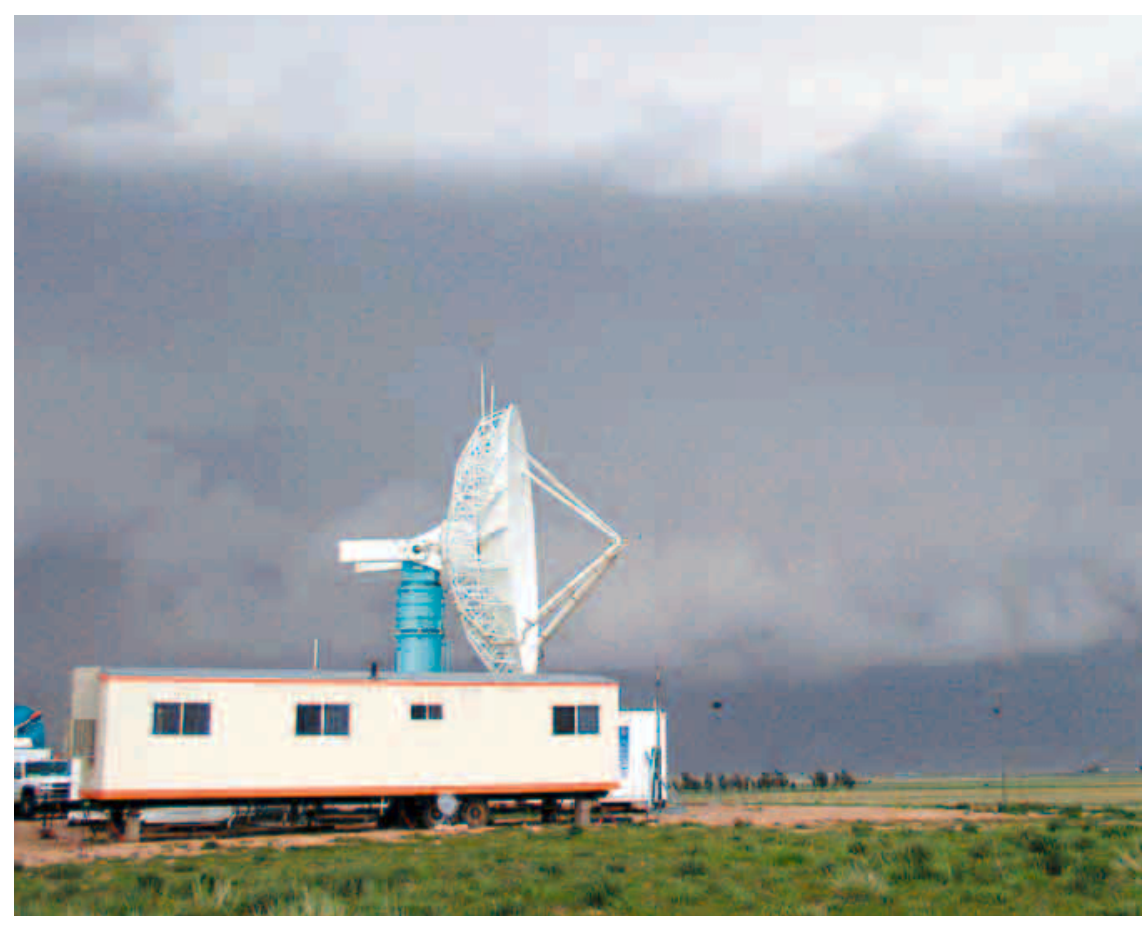

Le radar S-POL effectuant des mesures à l'approche d'un front le 15 juin 2002. S-POL est un radar Doppler, à double polarisation, en bande $S$ (mesure de l'indice de réfraction, classification des hydrométéores, taux de précipitation, vitesse radiale des écoulements en air clair). II est ici situé près de la ville de Balko dans le sud de I' «Oklahoma panhandle ». Sa portée en air clair comme nuageux est de 120 km environ.

En dépit des avancées régulières de la capacité des modèles numériques à prévoir de plus en plus de paramètres atmosphériques, la prévision précise des précipitations pendant les saisons chaudes reste encore un défi (Uccellini et al., 1994). Un point particulièrement bloquant pour la prévision numérique des précipitations reste la détermination correcte du déclenchement de la convection (à savoir : où ? et quand ?) et de son cycle de vie. Ces limitations s'expliquent en partie par les critères employés pour activer la convection, qui sont trop éloignés des mécanismes effectivement à l'œuvre. La prévision du déclenchement de la convection dans les modèles dépend fortement de la paramétrisation des processus de couche limite atmosphérique (CLA), mais également des évaluations très précises de la variabilité spatio-temporelle (4-D) du champ de vapeur d'eau dans la CLA et au- 
dessus (Crook, 1996). Récemment, Ducrocq et al. (2002) ont montré qu'un accroissement de la résolution ${ }^{(1)}$ dans les modèles, bien que permettant de s'affranchir de la paramétrisation de la convection profonde, n'améliore pas pour autant forcément la prévision des pluies. En général, il est nécessaire d'adjoindre une meilleure description de l'état initial du champ d'humidité, à l'échelle synoptique et à mésoéchelle, pour effectivement améliorer la prévision des précipitations. Ainsi, une caractérisation précise de la vapeur d'eau dans la basse atmosphère se révèle indispensable à la prévision quantitative des précipitations. Or la variabilité spatio-temporelle du champ de vapeur d'eau est très grande, notamment en raison du fait que :

- le champ de vapeur d'eau (contrairement au champ de température) n'est pas sujet aux processus d'ajustement de masse (la masse de la vapeur d'eau est égale à trois millièmes de celle de l'atmosphère) ;

- l'eau coexiste sous trois phases dans l'atmosphère. Par ailleurs, la vapeur d'eau est une variable atmosphérique essentielle mais qui reste encore difficilement mesurable, les concentrations de vapeur d'eau pouvant varier de trois ordres de grandeur dans la troposphère.

\section{Les difficultés de la mesure de la vapeur d'eau}

Alors que de nouveaux systèmes opérationnels, tels que les réseaux de radars et de profileurs de vent, sont déployés dans les grandes plaines des États-Unis et commencent à l'être en Europe, il n'existe pas d'équivalent permettant d'obtenir des profils verticaux précis de vapeur d'eau à la résolution verticale et temporelle requise (Weckwerth et al., 1999). Le fait que l'eau coexiste sous trois phases dans l'atmosphère complique également les stratégies d'observation. Les radiosondages ont longtemps été considérés comme l'outil de référence pour la caractérisation thermodynamique de l'atmosphère et constituent l'épine dorsale des systèmes opérationnels d'assimilation de données à des fins de prévision météorologique. Cependant, ces mesures souffrent d'un manque de représentati-

(1) La résolution utilisée est de l'ordre de $2 \mathrm{~km}$ sur l'horizontale, correspondant typiquement à celle des futurs modèles de prévision, par exemple le modèle Arome de Météo-France, qui sera opérationnel avant 2010.

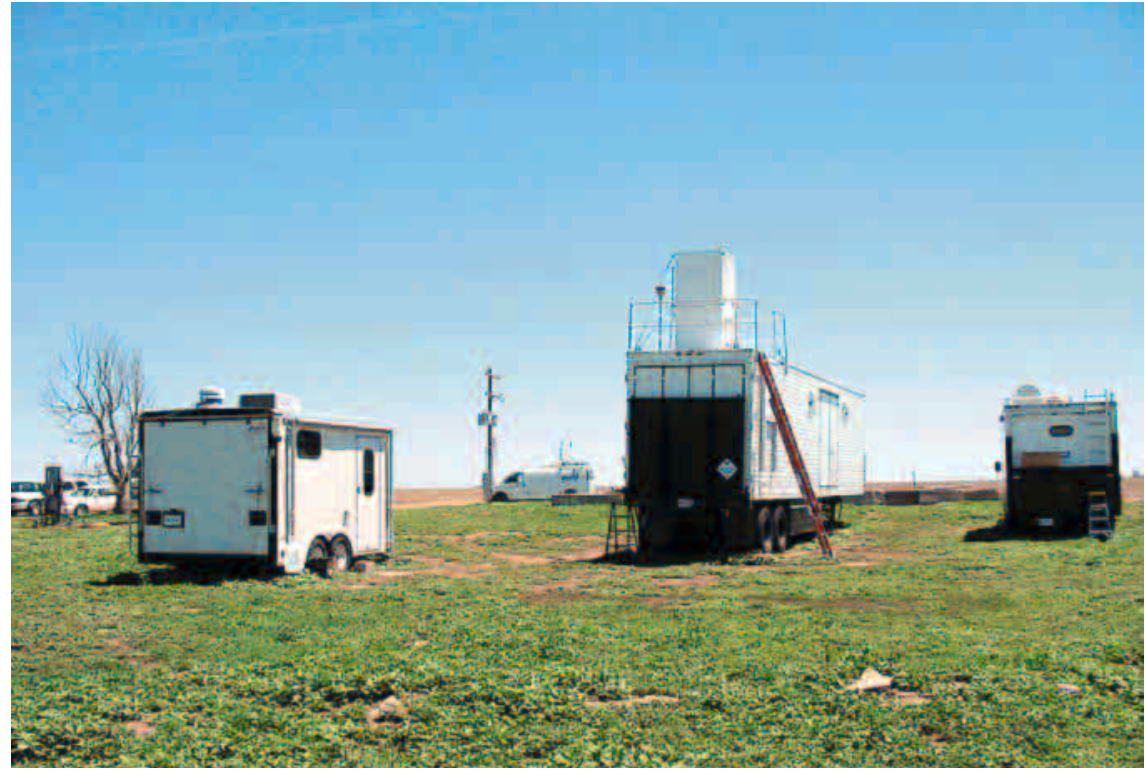

Les trois lidars du Laboratory for Atmospheres/Goddard Space Flight Center de la Nasa sur le site de Homestead, près du site de S-POL, dans I" "Oklahoma panhandle ». De gauche à droite : Harlie (lidar rétrodiffusion), SRL (lidar Raman pour la mesure du rapport de mélange de la vapeur d'eau) et Glow (lidar fonctionnant dans le proche ultraviolet pour la mesure du champ de vent)

vité et ne permettent pas une caractérisation 4-D suffisamment précise du champ de vapeur d'eau, ni du potentiel convectif associé (Weckwerth, 2000 ; Guichard et al., 2000) qui varie sur des échelles spatio-temporelles bien plus fines que le réseau actuel de radiosondages (maillage de l'ordre de quelques centaines de kilomètres et lâchers de ballons toutes les douze heures).

L'importance de la vapeur d'eau pour les études atmosphériques a conduit au développement de plusieurs techniques de télédétection permettant d'effectuer des mesures spatiales précises de cette vapeur d'eau dans l'atmosphère : radiomètres micro-ondes, lidars Raman ou à absorption différentielle, spectromètres infrarouges à transformée de Fourier, GPS (Global Positioning System) ou radar, par exemple. La variable mesurée dépend de la technique utilisée : rapport de mélange de la vapeur d'eau, humidité relative ou contenu intégré en vapeur d'eau. Enfin, la majorité des moyens de mesure cités ci-dessus sont des instruments de recherche développés pour répondre à une problématique scientifique particulière (CLA, précipitations, etc.). Ainsi, un instrument donné n'est-il pas nécessairement adapté à la caractérisation des différentes échelles spatio-temporelles des processus physiques intervenant dans les systèmes convectifs.

En dépit de l'intérêt évident d'une caractérisation simultanée de la vapeur d'eau à ces différentes échelles, il n'y avait pas eu jusqu'alors de tentative pour rassembler en un même endroit tous (ou presque) les instruments terrestres et aéroportés permettant de mesurer la variabilité de la vapeur d'eau atmosphérique dans une optique de modélisation et de prévision météorologique.

\section{Le projet IHOP 2002}

Dans ce contexte, les communautés scientifiques américaine et européenne intéressées ont défini le projet IHOP 2002 (International H2O Project). Cet article a pour but de présenter ce projet aux lecteurs de La Météorologie, mais aussi à la communauté française travaillant sur des objectifs proches de ceux de la campagne, mais non directement impliquée dans la phase terrain. Le lecteur se reportera au Plan d'opérations IHOP 2002 [www.atd.ucar.edu/ dir_off/projects/2002/IHOPdocs/ opsplan.doc] pour une description plus exhaustive du projet et au catalogue IHOP 2002 [www.joss.ucar.edu/ ihop/catalog/] pour le déroulement de la phase terrain.

Le projet IHOP 2002 (Weckwerth et al., 2003) a été lancé dès 1998, à la suite du « Lower-tropospheric water vapor workshop » (Weckwerth et al., 1999) organisé à Boulder, dans le Colorado, par le NCAR (National Center for Atmospheric Research) et la NOAA (National Oceanic and Atmospheric Administration). Cet atelier réunissait 


\section{Plates-formes et instruments impliqués dans IHOP 2002}

\begin{tabular}{|c|c|}
\hline Les avions & NRL P-3, UW King Air, Nasa Proteus, DLR Falcon, Nasa DC-8, Fll Learjet \\
\hline Les radars & $\begin{array}{l}\text { Vent : DOW, FM-CW radar, Xpow, Eldora, WCR } \\
\text { Taux de précipitation : S-POL, Smart-R, DOW, Xpow, Eldora }\end{array}$ \\
\hline Les lidars & $\begin{array}{l}\text { Vapeur d'eau : Leandre } 2 \text {, lidars à absorption différentielle du DLR, de la Nasa } \\
\text { et de la NOAA; lidar Raman de la Nasa } \\
\text { Vent : NOAA HRDL, Nasa Glow }\end{array}$ \\
\hline Télédétection & $\begin{array}{l}\text { Température et vapeur d'eau : Nasa-Nast, Nasa-HIS, Aeribago } \\
\text { (Interféromètres), UAH MIPS (radiomètre) } \\
\text { Contenu intégré en vapeur d'eau et eau liquide : GPS, DRI Mobile Radiometer }\end{array}$ \\
\hline In situ (PTUH) & $\begin{array}{l}\text { Profils : GPS dropsondes, Ncar Mlass, Ncar « reference sondes», } \\
\text { NSSL Mclass, Ncar Taos } \\
\text { Surface : NSSL Mesonet, Ncar ISFF }\end{array}$ \\
\hline $\begin{array}{l}\text { DLR : Deutsches } \\
\text { DRI : Desert Resє } \\
\text { NRL : Navy Resea }\end{array}$ & $\begin{array}{l}\text { NSSL : National Severe Storm Laboratory } \\
\text { UAH : University of Alabama Huntsville } \\
\text { UW : University of Wyoming }\end{array}$ \\
\hline
\end{tabular}

une vingtaine de scientifiques de divers horizons, tous concernés par le rôle de la vapeur d'eau en météorologie, ainsi que par les techniques de mesure associées. La phase terrain de IHOP 2002 s'est déroulée du 13 mai au 25 juin 2002 au-dessus des Grandes Plaines de l'Oklahoma (États-Unis). Les avantages de cette région sont multiples : de forts gradients d'humidité y sont fréquemment observés et l'activité convective y est intense. De plus, elle possède un réseau dense d'instruments, expérimentaux et opérationnels, déjà en place.

Les mécanismes d'initiation des systèmes convectifs y font souvent intervenir des lignes sèches, fréquentes dans cette région. Une ligne sèche est un gradient marqué d'humidité dans la CLA, large d'une dizaine de kilomètres, associé à une structure frontale séparant une masse d'air humide en provenance du golfe du Mexique et une masse d'air sec et plus chaud en provenance des Rocheuses. Sa longueur peut atteindre quelques centaines de kilomètres, voire plus. Dans la région des Grandes Plaines, la convection se déclenche souvent à moins de 10 kilomètres d'une ligne sèche (Bluestein et Parker, 1993).

Le projet IHOP 2002 a été financé par la NSF (National Science Foundation), la NOAA, la Nasa (National Aeronautics and Space Administration), le DOE (Department of Energy), le DOD (Department of Defense), le BBF (Bundesministerium für Bildung und
Forschung), le CNRS (Centre national de la recherche scientifique) et le CFCAS (Canadian Foundation for Climate and Atmospheric Sciences). La phase terrain a impliqué :

- environ 200 chercheurs (américains pour la plupart, français et allemands) ; - 50 plates-formes instrumentées (mobiles ou fixes) ;

- 6 avions équipés d'instruments de mesure in situ et de télédétection ;

- 2500 sondages en plus de ceux lâchés par le Service météorologique national ;

- un satellite dédié, Goes 11 ;

- 7 modèles de prévision à court et moyen terme.

Trente-six périodes d'observation intensive ont eu lieu pour un total de 268 heures de mesures de la vapeur d'eau sous toutes ses formes, ou presque.

Le projet IHOP 2002 s'articule autour de quatre séries d'objectifs complémentaires :

- La prévision quantitative des pluies.

Il s'agit de déterminer et de quantifier l'amélioration de la prévision des précipitations résultant d'une meilleure caractérisation du champ de vapeur d'eau.

- L'initiation de la convection.

L'objectif est de déterminer si, et comment, les mesures faites avant la formation des systèmes précipitants permettent d'améliorer la prévision de l'heure et de l'endroit du déclenchement de la convection.

- La couche limite atmosphérique.
Le but est de comprendre les relations entre l'humidité dans la CLA (et ses variations) et l'hétérogénéité du forçage à différentes échelles.

- L'instrumentation.

On cherche, d'une part, à définir la meilleure combinaison possible d'instruments de mesure de la vapeur d'eau permettant d'améliorer la prévision des précipitations et, d'autre part, à mieux quantifier le biais et la précision associés à ces instruments.

Pour atteindre ces objectifs, une stratégie couplée d'observation et de modélisation, impliquant des instruments in situ et de télédétection (au sol et aéroportés) et des outils de modélisation (prévisions à moyen et court terme, assimilation de mesures acquises pendant la campagne), a été mise en place. Cette stratégie, détaillée dans le Plan d'opérations IHOP 2002, est brièvement présentée ici.

\section{Domaine d'étude, dispositif expérimental et stratégie d'observation}

\section{Domaine d'étude et dispositif expérimental}

La figure 1 (page suivante) présente le domaine d'observation. La campagne était organisée à partir d'un centre d'opérations situé à Norman, Oklahoma, hébergé conjointement par le NSSL (National Severe Storm Laboratory) et le National Weather Service de la NOAA. Elle comportait quatre sites de coordination expérimentale : l'aéroport Will Rodgers (Oklahoma City) où étaient stationnés les six avions déployés pendant la campagne, Norman (Oklahoma) et Liberal (Kansas), sièges de la coordination de l'armada mobile (ensemble d'instruments mobiles, in situ et de télédétection), enfin Homestead, qui regroupait des instruments expérimentaux de télédétection terrestre (voir encadré ci-dessus pour la liste des instruments). Le déploiement de l'armada mobile répondait à un besoin de mesures continues depuis le sol, afin d'assurer un recoupement avec les mesures d'avions et une caractérisation de leur représentativité spatio-temporelle. 

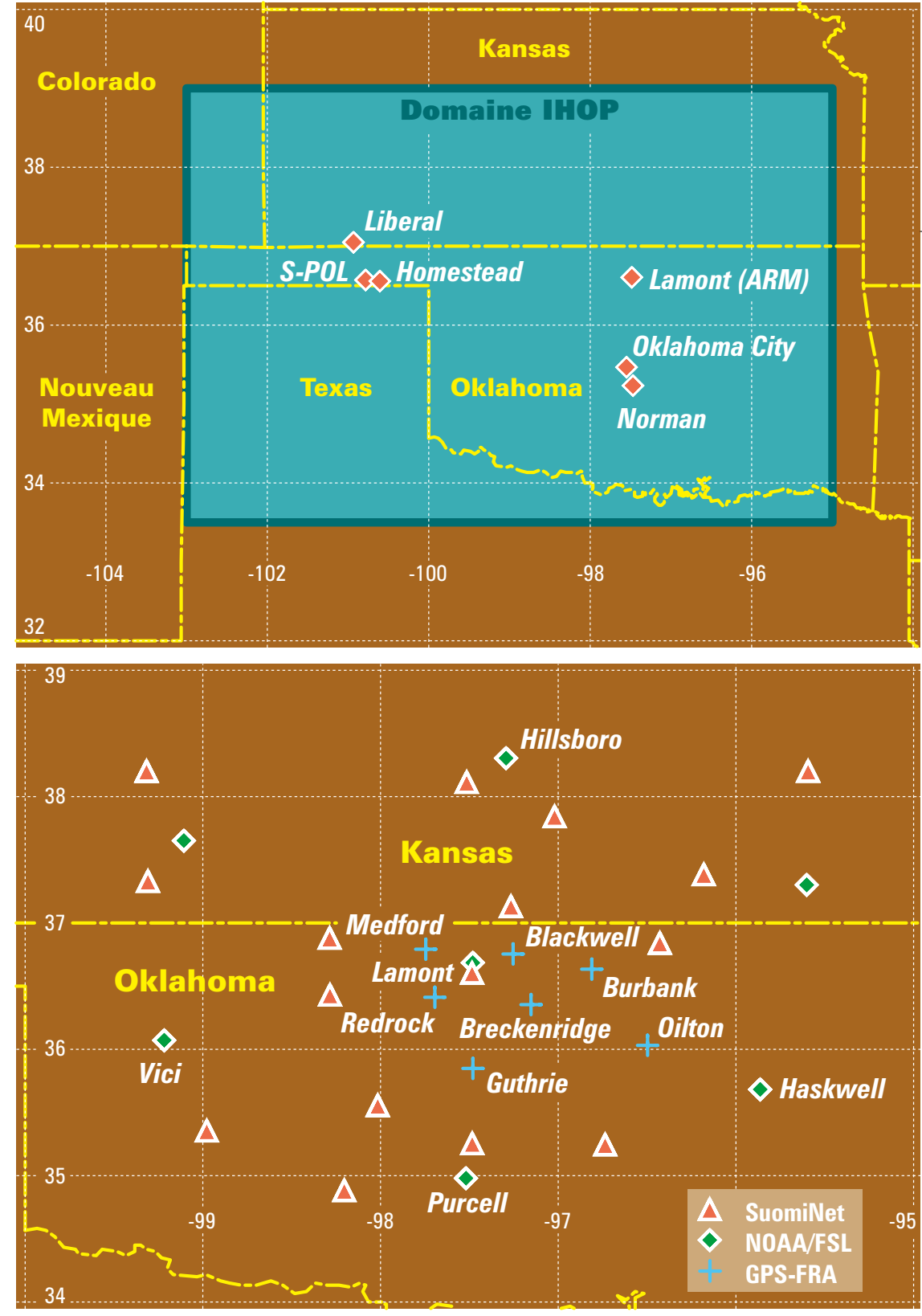

4 Figure 1 - Le domaine IHOP 2002 (rectangle bleu) et la situation géographique des principaux sites du dispositif expérimental.

V Figure 2 - Situation géographique des stations GPS dans le dispositif IHOP 2002 : 16 stations du Suominet (triangles rouges), 7 stations du FSL/NOAA

(losanges verts) et 7 stations de l'Insu (croix bleues). Les 25 stations du réseau dense ARM autour de Lamont ne sont pas indiquées.

Par ailleurs, l'État de l'Oklahoma possède plusieurs sites d'expérience, notamment un domaine ARM (Atmospheric Radiation Measurement) et plusieurs réseaux opérationnels, parmi lesquels le réseau très dense de stations météorologiques de surface Mesonet. À l'intérieur du domaine IHOP 2002 (figure 1), on recense également 29 sites hébergeant des profileurs de vent, le réseau de radars opérationnels Nexrad, 8 stations de sondage du réseau synoptique mondial, 23 stations de réception GPS mesurant le contenu intégré de vapeur d'eau (Doerflinger, 2001), espacées de 50 kilomètres et appartenant au réseau Suominet et au réseau de démonstration du FSL (Forecast System Laboratory) de la NOAA, plus 9 stations équipées pour mesurer l'humidité et la température du sol ainsi que les flux de surface $(3$ près de Homestead et 6 le long et au nord de la frontière séparant le Kansas de l'Oklahoma).

Le domaine ARM se compose d'un site central, Lamont, entouré de 4 sites périphériques, Hillsboro, Vici, Purcell et Haskwell (figure 2). Le site central possède un grand nombre d'instruments dédiés à la caractérisation des profils nuageux, de rayonnement et de paramètres d'état de l'atmosphère

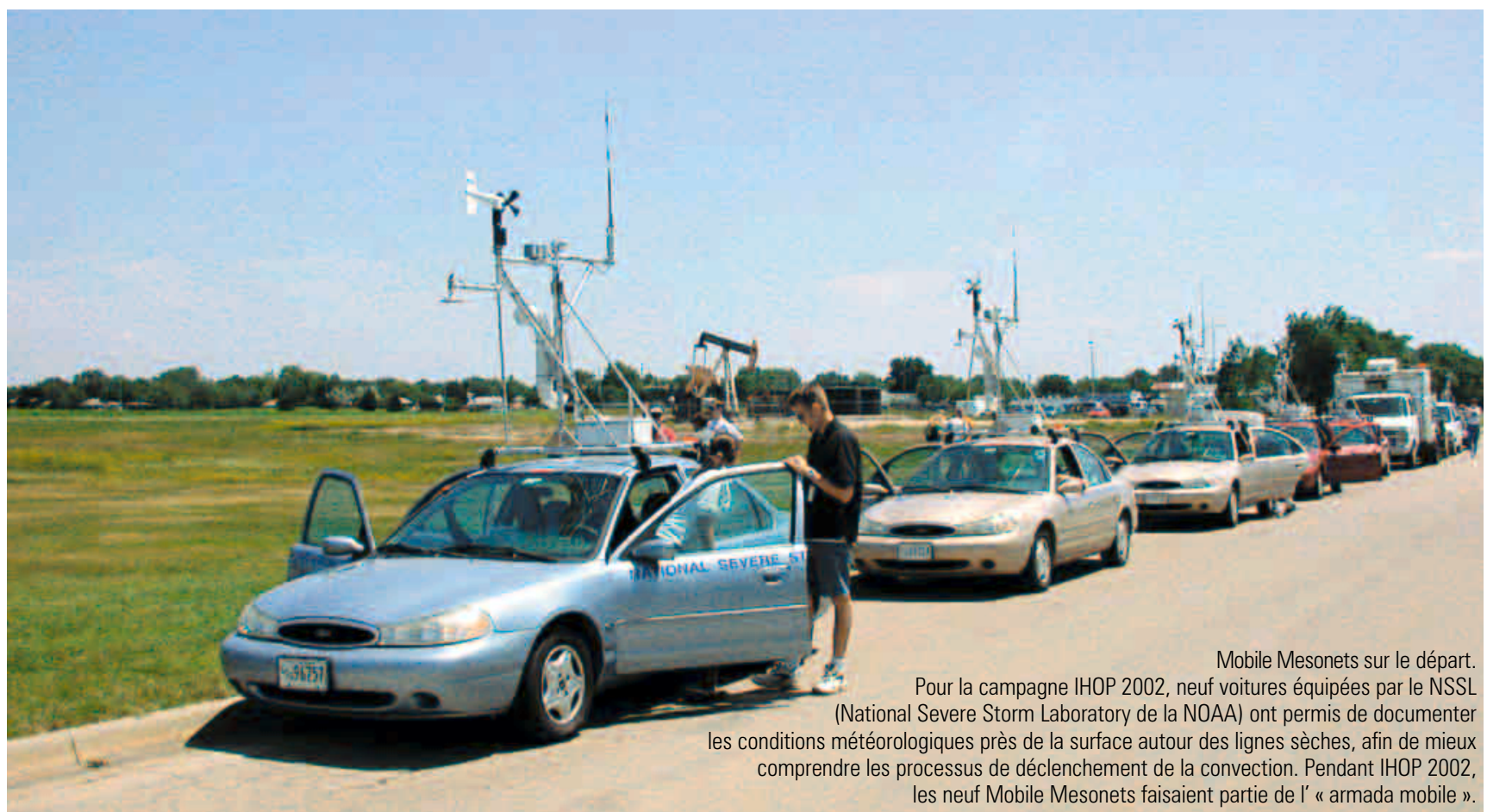


ainsi que de la structure du couvert. Autour de Lamont, 25 stations GPS mono-fréquence sont réparties sur une surface inférieure à $40 \mathrm{~km}^{2}$ (réseau dense L1), afin de calculer le champ 4-D de vapeur d'eau par tomographie GPS.

\section{Stratégie d'observation}

Pour répondre aux objectifs du projet IHOP 2002, la stratégie d'observation a été définie de manière à utiliser les différents moyens d'observation de façon complémentaire. Cinq types d'expériences, faisant intervenir à divers degrés avions, armada mobile et moyens terrestres, ont été prévus et mis en ouvre pendant la campagne (les détails concernant la conception de ces expériences sont donnés dans le Plan d'opérations IHOP 2002) :

- Expériences BLE (Boundary Layer Evolution) : l'objectif est de déterminer l'évolution de la distribution horizontale et verticale de l'humidité dans la CLA au cours des premières heures suivant le lever du soleil. Ces expériences permettront également d'analyser la représentativité des sondages réalisés à 12 h UTC ( 7 heures locales), ces sondages jouant un rôle dans la prévision à court terme du déclenchement de la convection puisqu'ils sont utilisés pour estimer la stabilité atmosphérique et la probabilité de déclenchement de la convection.

- Expériences BLH (Boundary Layer Hydrology) : elles concernent l'analyse de la variabilité de la vapeur d'eau dans la couche limite. Il s'agit d'expliquer comment se développent les hétérogénéités de la vapeur d'eau dans la CLA en relation avec l'humidité et l'occupation des sols, l'orographie et le forçage atmosphérique, ainsi que de caractériser le bilan de vapeur d'eau et de comprendre l'évolution spatio-temporelle de l'humidité dans la CLA et au-dessus.

- Expériences CI (Convection Initiation) : l'objectif est de caractériser la variabilité de la vapeur d'eau ainsi que la dynamique le long des zones de convergence dans la CLA, en relation avec le déclenchement de la convection. - Expériences LLJ (Low-Level Jet) ${ }^{(1)}$ : l'objectif est de comprendre le déclenchement de la convection (pendant la journée) et l'alimentation des systèmes convectifs de mésoéchelle déjà

(1) On a regroupé ici les expériences evening $L L J(E L L J)$ et morning $L L J(M L L J)$.

(2) Du norvégien " bora ", terme désignant un phénomène de type mascaret dans les fjords norvégiens. Le bore correspond à la notion mathématique de soliton.

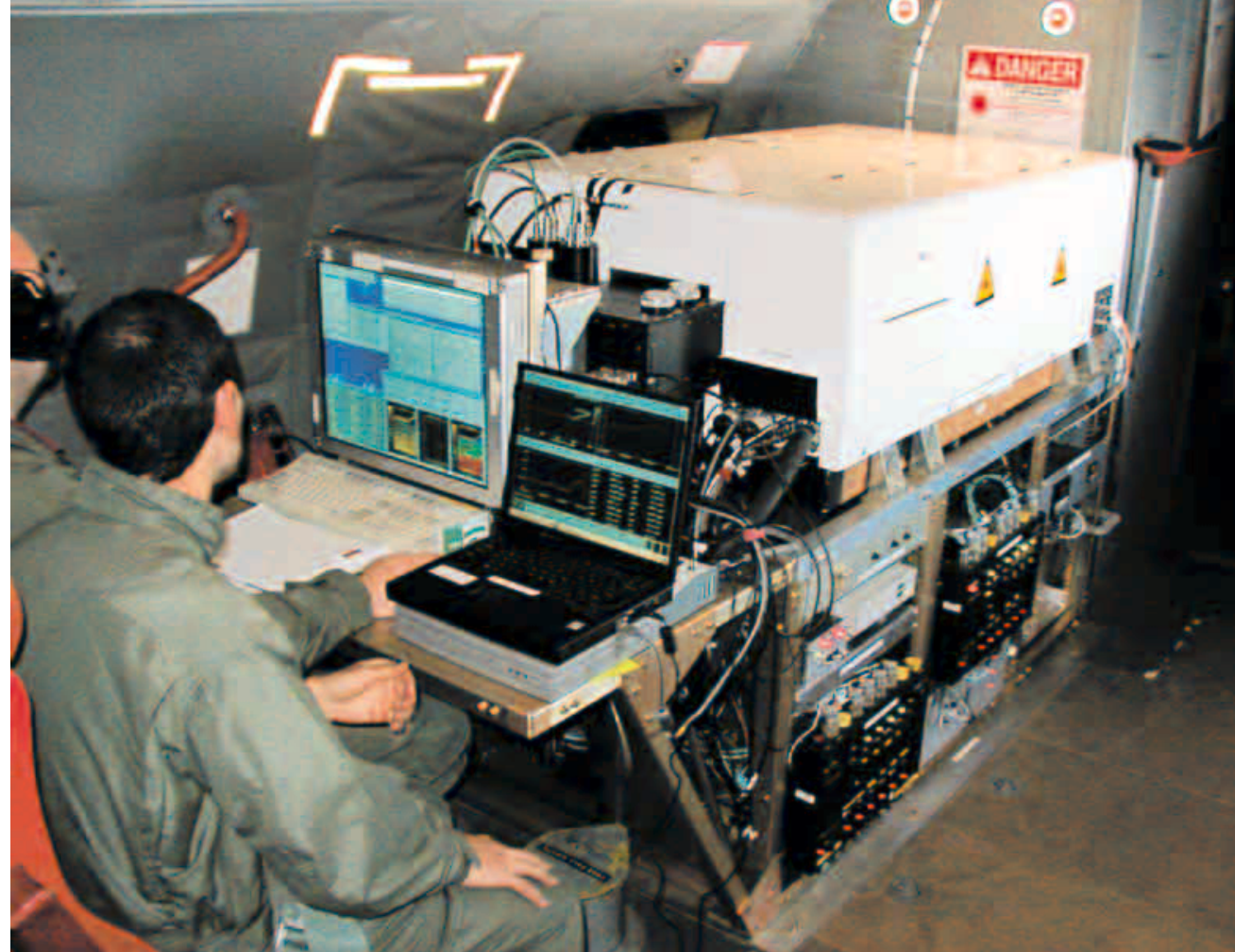

Nadir Amarouche (Division technique de l'Insu) devant les écrans de contrôle du lidar Leandre 2, face à l'arrière de l'avion $P$-3. Le faisceau laser est émis dans l'atmosphère au travers d'une fenêtre située à gauche du système (le sommet de celle-ci est visible au-dessus du capot blanc).

formés (pendant la nuit), en relation avec l'évolution, en amont, de la distribution de la vapeur d'eau associée aux jets de basse couche (low-level jets) en provenance du golfe du Mexique.

- Expériences Bore : l'objectif est de comprendre le déclenchement des phénomènes ondulatoires de type bore $^{(2)}$ en relation avec les courants de densité émanant de systèmes convectifs de mésoéchelle au cours de la nuit et leur rôle dans le maintien de la convection.

Pendant la campagne, les missions étaient préparées en étroite collaboration avec les prévisionnistes. Chaque jour, sur la base des prévisions à court et moyen terme, des propositions étaient faites par les scientifiques pour les jours $\mathrm{J}+1$ et $\mathrm{J}+2$. Les missions du jour $\mathrm{J}$ étaient finalisées environ quatre à cinq heures avant l'heure prévue du décollage des avions impliqués. Pendant les missions, les avions et l'armada mobile étaient tenus informés par le centre des opérations de l'évolution des conditions météorologiques en temps réel, sur la base d'images satellitaires et de prévisions à très court terme. Les modèles de prévision en temps réel ainsi que les modalités du support en termes de prévision à moyen et à court terme sont détaillés dans le Plan d'opérations IHOP 2002.

\section{La contribution française}

La contribution française au projet IHOP 2002 s'articule autour de trois équipes de l'Institut Pierre-Simon Laplace et du Centre national de recherches météorologiques :

- L'équipe dite Leandre 2, dont le thème de recherche et l'expertise sont centrés sur les processus de CLA et d'échange de matière, d'énergie et d'humidité entre la surface et l'atmosphère, dans des conditions d'air clair ou nuageux. L'équipe a activement contribué à la phase terrain d'IHOP 2002, avec la mise en œuvre des mesures de rapport de mélange de vapeur d'eau dans la troposphère effectuées par le lidar à absorption différentielle Leandre 2 (Bruneau et al., 2001, voir encadré page suivante). À bord du NRL $P-3$, Leandre 2 a effectué environ 140 heures de vols scientifiques, réparties comme suit : 7 missions BLH pour un total de 35 heures, 3 missions BLE pour un total de 21 heures, 12 missions CI pour un total de 73 heures et 2 missions ELLJ pour un total de 11 heures. Pour la première fois, des mesures de vapeur d'eau en visée horizontale dans la CLA ont été effectuées.

- L'équipe dite GPS, dont l'expertise est centrée sur la physique du signal GPS, de son analyse et de l'interprétation des mesures atmosphériques 
déduites. L'équipe GPS a aussi participé à l'expérience via la mise en œuvre de 7 stations GPS (Blackwell, Breckenridge, Burbank, Guthrie, Medford, Oilton, Redrock) qui sont venues étoffer les réseaux existants sur les plaines du « Mid-west » américain (figure 2). Les quatre premières stations ont été déployées afin d'encadrer au plus près le site central ARM multiinstruments, mais aussi le réseau dense L1. Les trois autres stations GPS ont permis de densifier les réseaux de grande échelle de l'Ucar/SuomiNet et de la NOAA/FSL, mais aussi d'homogénéiser la distribution spatiale au sudest du site ARM (maillage de l'ordre de $100 \mathrm{~km}$ ).

- L'équipe dite Modélisation, dont les thèmes de recherche concernent l'étude des processus de convection nuageuse et leur paramétrisation à partir d'une approche reposant sur la modélisation explicite des nuages (à l'échelle de quelques dizaines de mètres jusqu'au kilomètre). Pendant la campagne IHOP 2002, l'un des membres de cette équipe a également participé à l'effort de modélisation opérationnelle impliquant le modèle mésoéchelle à aire limitée MM5 [www.mmm.ucar.edu/mm5/ mm5-home.html]. Pendant IHOP 2002, le calcul fonctionnait avec trois modèles emboîtés de résolution croissante, de $12 \mathrm{~km}$ à $1,5 \mathrm{~km}$ pour le plus petit domaine centré sur le site d'Homestead. Pour la première fois, les profils de température et d'humidité entre 0 et $3 \mathrm{~km}$ d'altitude, mesurés par les interféromètres Aeri localisés sur 6 sites du domaine IHOP 2002, ont été assimilés.

Compte tenu de la très parcellaire connaissance actuelle, la richesse des données collectées au cours de l'expérience va réellement permettre d'en savoir beaucoup plus sur les modes de variabilité du champ de vapeur d'eau à échelle fine. Il reste à élucider de nombreuses questions concernant les causes et les conséquences de cette variabilité, son impact sur le fonctionnement de la couche limite et le développement de la convection nuageuse. Pour aborder ces différents sujets, la communauté française s'est structurée autour de trois thèmes scientifiques transverses aux équipes :

- Processus de CLA et variabilité du champ de vapeur d'eau. L'objectif principal est de déterminer l'influence de la surface, de son hétérogénéité, du type d'organisation dans la CLA (par exemple, rouleaux de convection) et du processus d'entraînement sur la variabilité du champ de vapeur d'eau dans la CLA (identifier et quantifier les sources de variabilité).

- Impact des hétérogénéités de surface sur l'initiation de la convection, effets de brise. L'objectif principal est de comprendre le rôle de la surface dans le déclenchement de la convection et l'impact de la forme de la transition séparant les surfaces de nature différente induisant un chauffage différentiel, soit notamment ici la frontière mésoéchelle entre les zones arides à l'ouest et celles plus humides, couvertes de végétation, à l'est de la région de l'expérience.

- Initiation et entretien de la convection. L'objectif principal est de mieux comprendre pourquoi la convection se déclenche ou non, comment les systèmes convectifs sont alimentés en humidité, en relation avec les deux thèmes précédents.
Par ailleurs, un certain nombre d'objectifs méthodologiques ont été définis, en particulier concernant l'analyse des délais obliques et la tomographie par GPS. Les objectifs scientifiques, quant à eux, s'articulent autour de l'étude de la dynamique et de la variabilité de la vapeur d'eau révélée par le GPS, dans le cadre de l'analyse des précurseurs à la convection, de l'analyse tomographique à l'échelle locale (réseau L1) et synoptique (réseau Suominet) et de l'assimilation des données GPS dans les modèles de prévision. La confrontation des simulations avec ces résultats permettra aussi une évaluation sans précédent de la qualité des champs de vapeur d'eau calculés par les modèles, ainsi qu'une analyse de la validité des mécanismes impliqués dans l'initiation de la convection modélisée.

\section{Le lidar Leandre 2}

Le lidar aéroporté Leandre 2 est un lidar à absorption différentielle permettant de mesurer le rapport de mélange de vapeur d'eau dans la troposphère avec une résolution temporelle et spatiale (le long de la ligne de visée) de l'ordre de la seconde et de la dizaine de mètres respectivement (les résolutions effectives dépendent de la précision souhaitée sur la mesure). Le principe est simple : deux rayonnements sont émis simultanément ou presque, sur deux longueurs d'onde très proches l'une de l'autre, dont l'une coïncide avec une bande d'absorption de la vapeur d'eau dans la région 727-770 nm. À une distance donnée, le rapport entre les puissances reçues est proportionnel à l'absorption différentielle entre les deux voies. Celle-ci est fonction de la densité des cibles et de leur pouvoir absorbant, lui-même proportionnel à la température. Il est alors possible de remonter à la densité des cibles, c'est-à-dire des molécules d'eau, et par voie de conséquence au rapport de mélange de vapeur d'eau. Leandre 2 (Bruneau et al., 2001) a été développé à l'Institut Pierre-Simon Laplace (IPSL) avec le soutien de la Division technique de I'Institut national des sciences de l'univers (Insu) et du Centre national d'études spatiales (Cnes). II est opérationnel depuis 1995. Leandre 2 utilise une source laser de type Alexandrite. La longueur d'onde d'opération peut être ajustée en fonction des conditions d'humidité (choix de sept longueurs d'onde prédéfinies). De 1995 à 2001, Leandre 2 a contribué à plusieurs projets internationaux à bord de l'Avion de télédétection et de recherche atmosphérique (Arat) exploité par l'Institut géographique national (IGN), I'Insu, le Cnes et Météo-France.

Dans le cadre du projet IHOP, sa contribution ayant été jugée primordiale par l'ensemble des participants, Leandre 2 a été intégré dans un P-3 de la Navy Research Laboratory (NRL). Cette intégration a été menée conjointement par la Division technique de l'Insu et par l'Atmospheric Technology Division (ATD) du NCAR (Keeler et al., 2002). Dans le cadre de l'intégration, certaines modifications ont été apportées qui en ont fait un instrument unique et ont rendu sa contribution plus importante encore : à bord du P-3, Leandre 2 a désormais la capacité de viser, non seulement verticalement (au nadir et au zénith, comme à bord de l'Arat), mais également horizontalement, cette dernière possibilité étant particulièrement attrayante pour des vols effectués dans la CLA. La grande première au cours de IHOP a donc consisté à coupler les mesures de rapport de mélange de vapeur d'eau "plus classiques effectuées dans un plan vertical par les systèmes Dial du DLR et de la Nasa avec les mesures horizontales effectuées par Leandre 2 afin de caractériser le champ 3-D de vapeur d'eau, ce qui est en soit un des objectifs de IHOP. Cette capacité à viser horizontalement fait que Leandre 2 est considéré par la communauté IHOP comme un instrument clé (avec le radar Eldora) pour la thématique $\mathrm{CI}$ (initiation de la convection). En effet, la visée horizontale a permis de caractériser l'hétérogénéité horizontale du champ de vapeur d'eau alimentant la convection, tout en s'affranchissant des problèmes généralement liés à la présence de nuages puisque ceux-ci ne sont pas échantillonnés. C'est ainsi un excellent moyen de détection des lignes sèches le long desquelles se déclenche la convection et qui sont au cœur de la problématique $\mathrm{Cl}$. 


\section{Premiers résultats}

L'expérience s'étant déroulée récemment, il est encore trop tôt pour présenter des résultats scientifiques élaborés et pour faire un bilan. Dans cette section, nous avons choisi d'illustrer les études numériques en cours sur la sensibilité du déclenchement de la convection aux conditions initiales d'humidité dans l'atmosphère. Une première analyse des observations Leandre 2 effectuée au cours de la mission ELLJ du 20 juin pendant IHOP 2002 est également présentée. Ce seul exemple montre déjà toute la complexité des structures de fine échelle caractérisant la variabilité de la vapeur d'eau et des mécanismes impliqués dans l'initiation et le maintien de la convection.

\section{Expériences numériques de sensibilité du déclenchement de la convection}

Plusieurs études soulignent la grande sensibilité de l'initiation de la convection au champ d'humidité dans les basses couches (Crook, 1996 ; Weckwerth, 2000). Cette sensibilité est illustrée ici par trois simulations académiques de convection profonde diurne sur le site ARM des Grandes Plaines américaines (cas d'étude du projet européen Eurocs, voir aussi Xu et al., 2002). Ces simulations ont toutes été réalisées avec le modèle communautaire Méso-NH (Lafore et al., 1998) en mode explicite avec une résolution horizontale de $2 \mathrm{~km}$. Elles diffèrent seulement par le champ d'humidité initial utilisé. Dans l'expérience de référence, les conditions initiales correspondent à un profil de rapport de mélange issu de sondages ballon. Dans la première (deuxième) expérience de sensibilité, ce profil est humidifié (asséché) de 1 g. $\mathrm{kg}^{-1}$ dans les basses couches de l'atmosphère. L'écart obtenu entre les deux expériences est ainsi comparable aux incertitudes actuelles sur la mesure de la vapeur d'eau. L'évolution du champ de nuages est globalement similaire pour les trois simulations (figure 3 ). On note pour chacune une succession de phases : initialement un ciel sans nuages ; puis une période de convection peu profonde, avec des sommets de nuages ne dépassant pas 2 kilomètres ; et enfin, dans l'après-midi, une phase de convection plus pro-

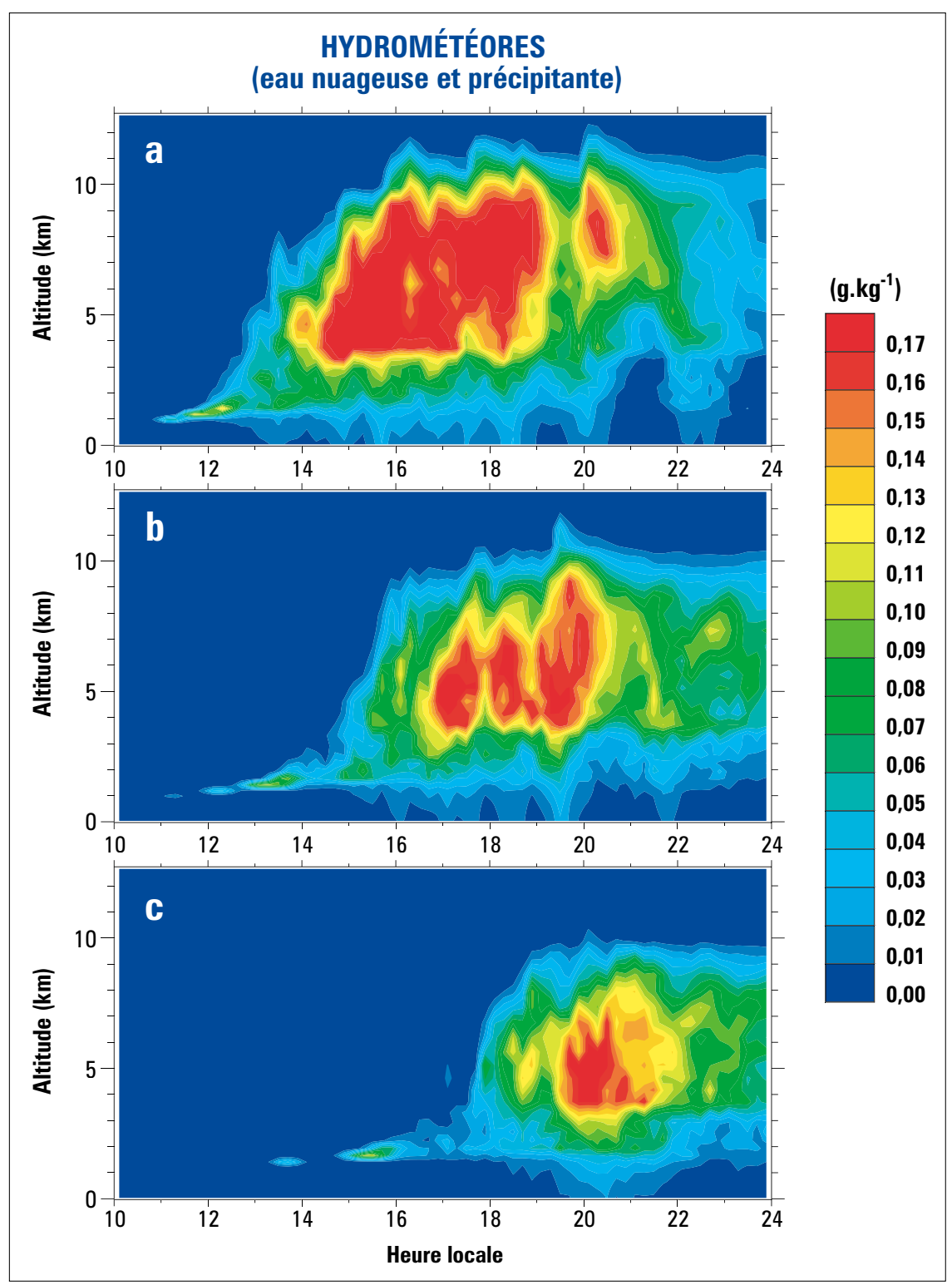

Figure 3 - Évolution temporelle du contenu total en hydrométéores (eau nuageuse et précipitante) pour trois simulations explicites de nuages différant par le profil initial de vapeur d'eau dans les basses couches atmosphériques : I'expérience de référence initialisée à partir d'un profil obtenu par sondage ballon (b), l'expérience de sensibilité avec humidification des basses couches (a) et l'expérience de sensibilité avec assèchement des basses couches (c).

fonde, accompagnée de précipitations, avec des sommets de nuages à plus de 10 kilomètres d'altitude (les plages bleu clair qui s'étendent, sur la figure 3 , jusqu'à la surface correspondent à la pluie atteignant le sol).

Cependant, des différences notables apparaissent aussi entre ces trois simulations. Plus le profil initial est humide dans les basses couches, plus les nuages sont épais et les pluies importantes. De plus, la succession des trois phases - ciel clair, petits cumulus, cumulonimbus - est bien distincte pour chacune des simulations, avec des écarts pouvant atteindre plusieurs heures. Les premiers nuages bas commencent à se développer avec deux heures de retard pour la simulation la plus sèche (figure 3c) par rapport à la simulation la plus humide (figure $3 \mathrm{a}$ ). La durée de cette phase est également variable : plus le profil initial est humide, plus elle est courte. Le décalage temporel et la durée de la phase de convection précipitante sont tout aussi significatifs. Ces différences s'expliquent par plusieurs mécanismes, incluant une diminution de l'inhibition convective lorsque les basses couches s'humidifient et aussi, très probablement, une évaporation-dilution des nuages bas plus forte lorsque l'atmosphère s'assèche. Cette étude de sensibilité montre clairement la difficulté de prévoir correctement l'activité convective diurne, en termes d'initiation comme de durée, lorsque le champ de vapeur d'eau n'est pas suffisamment bien connu. 


\section{Alimentation \\ d'un système convectif \\ nocturne sur les Grandes Plaines : le cas du 20 juin 2002}

Dans la journée du 19 juin 2002, la convection profonde s'est développée le long d'un front froid quasi-stationnaire orienté sud-ouest - nord-est, s'étendant depuis le Colorado jusqu'au $\mathrm{Ne}$ braska. Les champs d'énergie potentielle disponible pour la convection (Cape) prévus par les modèles indiquaient des conditions favorables au déclenchement de la convection le long de ce front. Les premières tourelles convectives firent leur apparition dans le sud-est du Colorado vers $20 \mathrm{~h}$ UTC. À partir de ce moment, la convection s'est déclenchée de manière séquentielle le long du front, depuis son extrémité sud jusqu'à son extrémité nord (vers 23 h UTC).

La mission ELLJ de la nuit du 19 au 20 juin 2002 avait pour objectif d'analyser les mécanismes d'alimentation en humidité des systèmes convectifs formés au cours de la journée du 19 juin 2002, au nord de l'Oklahoma panhandle ${ }^{(1)}$. La figure 4 montre la prévision à court terme des champs de vent et de précipitations fournie par le modèle RUC du NOAA/FSL (résolution horizontale de $20 \mathrm{~km}$ ) pour $3 \mathrm{~h}$ UTC le 20 juin. Le front s'étend alors depuis le Colorado jusqu'au Nebraska et est caractérisé par un fort gradient de vent à $850 \mathrm{hPa}$ (en direction essentiellement) et par de fortes précipitations. La figure 4 indique également que l'alimentation en humidité des systèmes convectifs au nord de l' «Oklahoma panhandle » (juste à l'est de la frontière entre le Colorado et le Kansas) se fait via un jet de basses couches $(22-25 \mathrm{~m} / \mathrm{s})$ en provenance du golfe du Mexique.

(1) La bande étroite de cet État, située au nord, tout à l'ouest. Elle doit sont nom à sa forme géographique en "queue de casserole».

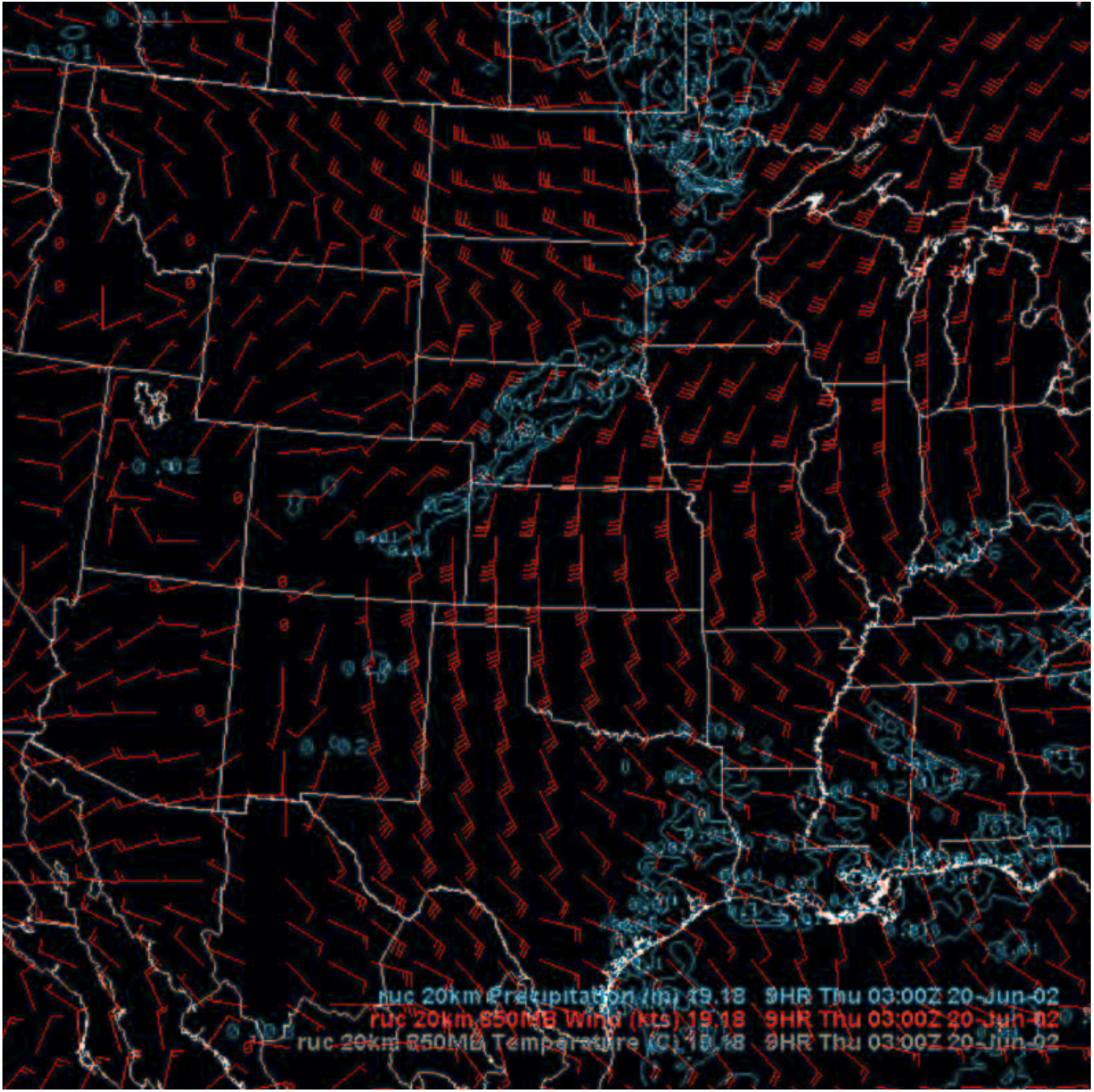

Figure 4 - Champs de vent et de précipitations (en bleu) prévus par le modèle RUC du NOAA/FSL (résolution horizontale de $20 \mathrm{~km}$ ) pour $3 \mathrm{~h}$ UTC le 20 juin.

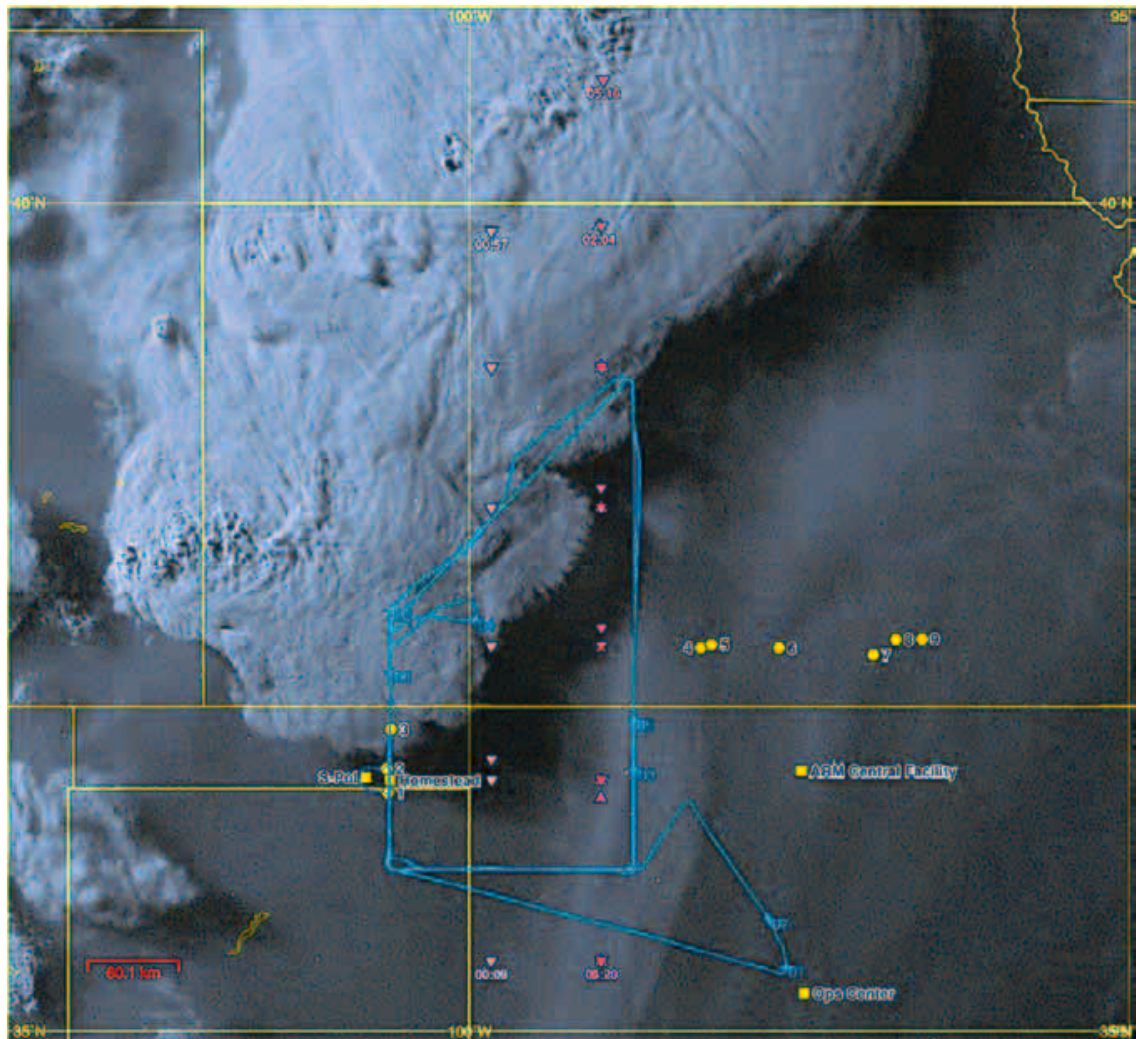

Figure 5 - Trajectoire du NRL P-3 (ligne bleue) au cours de la mission ELLJ du 20 juin 2002 superposée à l'image visible Goes-8 de $1 \mathrm{~h} 03$ UTC. Les cercles jaunes sont les stations au sol du NCAR, les triangles les points de lâchers de dropsondes. 


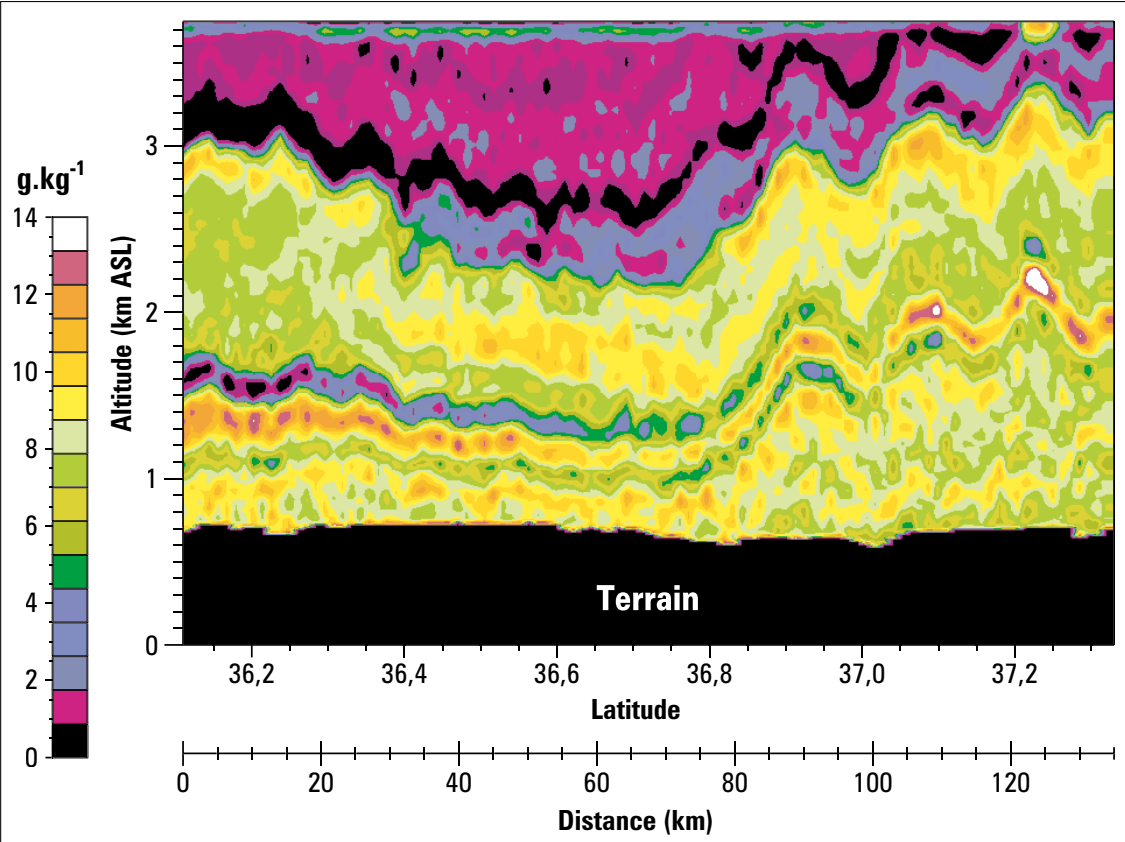

Figure 6 - Coupe verticale du rapport de mélange de la vapeur d'eau mesurée par Leandre 2 (visée au nadir) entre 4 h 10 et 4 h 27 UTC le 20 juin 2002.

Pour cette mission, deux avions étaient impliqués entre $23 \mathrm{~h}$ UTC le 19 juin et 6 h UTC le 20 juin 2002 : le NRL $P$-3 (avec à son bord Leandre 2 en visée au nadir et Eldora) et le LearJet dédié au lâcher de dropsondes (figure 5).

$\mathrm{Au}$ sud du système convectif, alors que le $P$-3 remonte vers le nord, les mesures de rapport de mélange de vapeur d'eau effectuées par Leandre 2 ont mis en évidence la structure complexe du champ d'humidité dans les basses couches : le jet de basses couches en provenance du golfe du Mexique, observé près de la surface (épaisseur de $700 \mathrm{~m}$ au sud), était surmonté d'une couche humide (entre 1,8 et $3 \mathrm{~km}$ ), s'apparentant à une couche résiduelle (CLA fossile de la veille, fraîchement découplée par le développement d'une couche stable à la surface après le coucher du soleil). Une couche très mince et très sèche séparait les deux couches humides (un phénomène fréquemment observé dans les Grandes Plaines).

Plus au nord, Leandre 2 a mis en évidence le brusque épaississement du jet de basses couches (de 400 à $800 \mathrm{~m}$ ) associé à une structure ondulatoire (figure 6). La couche humide d'altitude a eu un comportement similaire. Ce phénomène est apparenté à un bore atmosphérique. Les images radar du réseau Nexrad semblent indiquer que ce bore a été déclenché par un front de surface lié à un courant de gravité émanant du système convectif vers

1 h 00 UTC. Ce bore d'une intensité exceptionnelle (également vu par SPOL) a été échantillonné quatre fois par Leandre 2 sur l'axe nord-sud le plus à l'ouest. L'évolution temporelle du phénomène a ainsi pu être mise en évidence, notamment la formation progressive de cumulus au sommet de la couche humide en altitude. L'analyse de l'ensemble des cas de est superposé. bores atmosphériques échantillonnés pendant IHOP 2002 (12 cas en tout, le cas du 20 juin 2002 ayant été le seul à être échantillonné par un moyen de télédétection laser aéroporté en plus des moyens de télédétection au sol et des mesures in situ aéroportées) suggère que les phénomènes de ce type jouent un rôle crucial dans le maintien de la convection nocturne, en favorisant la formation de nuages en amont des systèmes convectifs déjà matures. $\mathrm{Si}$ cette conclusion se révèle exacte, une simulation correcte de ces bores semble indispensable.

La figure 7 montre le rapport de mélange de vapeur d'eau simulé par MM5 le long de l'axe sud-nord parcouru par le $N R L P$-3. La simulation a été effectuée sur un domaine de 1000 km de côté centré sur le radar S-POL, avec une résolution de $12 \mathrm{~km}$. Les conditions aux limites étaient fournies par les prévisions du modèle ETA (Unisys Weather, http://weather. unisys.com/eta).

Le modèle est capable de reproduire l'épaississement de la couche humide (CLA et couche résiduelle) près du système convectif au nord (ce système était bien prédit par le modèle). Les valeurs de rapport de mélange simulées dans la partie supérieure de la couche d'humidité sont aussi en bon accord avec

Figure 7 - Coupe verticale du rapport de mélange de la vapeur d'eau simulée par MM5 avec une résolution de $12 \mathrm{~km}$ sur l'axe documenté par Leandre 2 dans la figure 6 , à 5 h UTC le 20 juin 2002. Le champ de vent horizontal

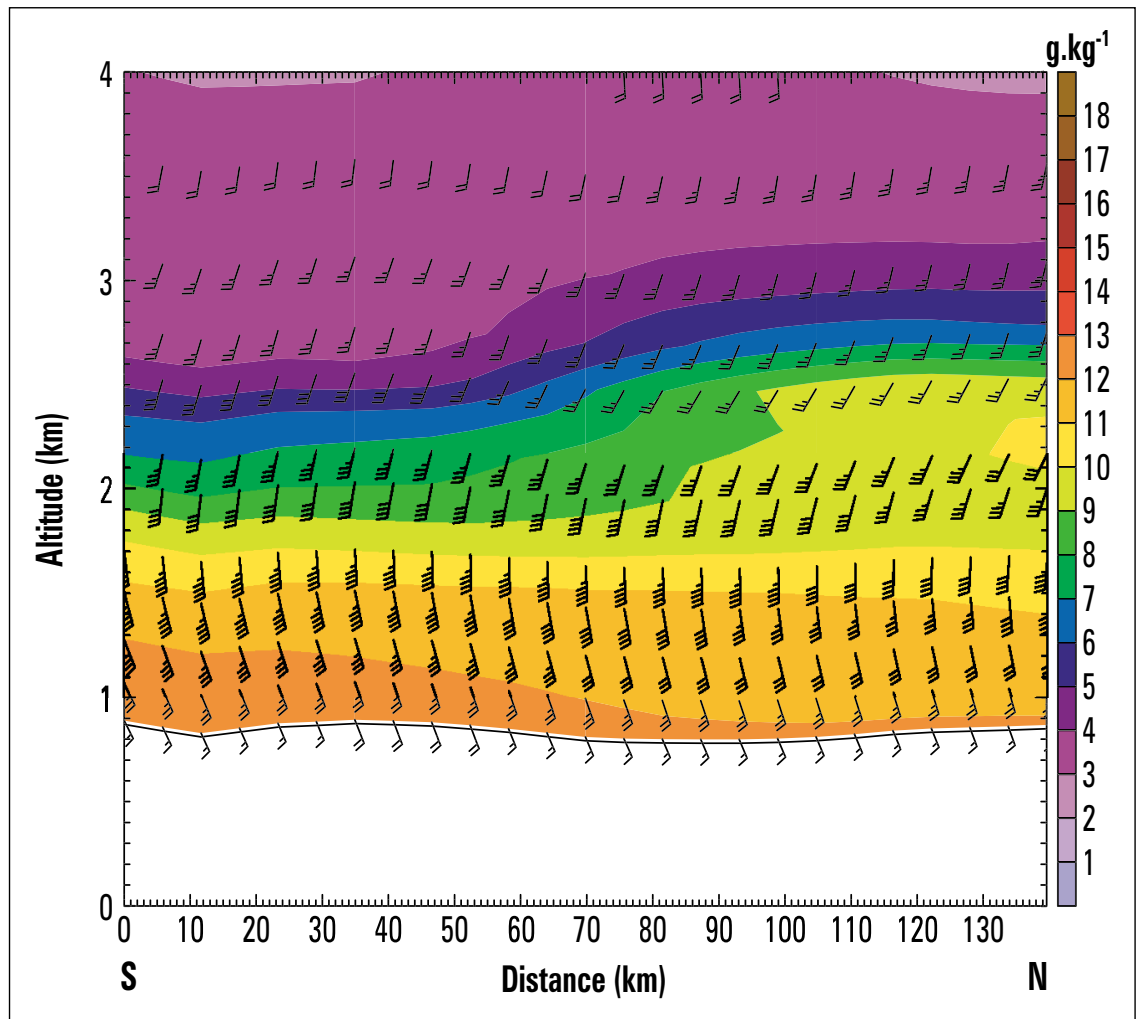


celles mesurées par Leandre 2. Un certain nombre de différences entre simulation et observations lidar sont cependant notables :

- L'absence d'ondes au sommet de la CLA et de la couche résiduelle. Cela est vraisemblablement lié à la résolution de la simulation $(12 \mathrm{~km})$, ne permettant pas de résoudre ces mouvements ondulatoires.

- L'absence de la couche très sèche et très mince (100 m d'épaisseur au plus) au sommet de la CLA (également observée sur les sondages ballon), à l'interface entre la CLA et la couche résiduelle. Là encore, la résolution verticale utilisée ne permet que très marginalement de reproduire ce type de structure.

- L'épaisseur plus faible (1 km par endroits) de la couche humide, couche qui est aussi dans sa partie inférieure plus humide d'environ $3 \mathrm{~g} . \mathrm{kg}^{-1}$ : de nombreux facteurs participent probablement à ces erreurs, incluant les conditions aux limites utilisées, qui sont des prévisions du modèle ETA et non des analyses, ainsi que la physique du modèle (en particulier la turbulence).

Cette comparaison illustre les difficultés associées à la simulation des événements convectifs nocturnes qu'il conviendra de résoudre afin de répondre aux objectifs du programme IHOP 2002.

\section{Conclusions}

Les objectifs du projet IHOP 2002 s'inscrivent dans les priorités de nombreux programmes nationaux et internationaux (Eurocs et Gewex). IHOP 2002 est un projet unique de par l'ampleur du volet instrumental et du volet modélisation-prévision mis en oeuvre. Parmi les avancées les plus significatives auxquelles devrait contribuer IHOP 2002, on peut citer :

- la meilleure quantification du biais et de la précision associés aux différents instruments mesurant la vapeur d'eau ;

- la meilleure compréhension des processus (y compris les interactions d'échelles) intervenant dans le déclenchement de la convection profonde en relation avec la CLA et la surface ;

- l'amélioration de l'analyse de l'humidité et du cycle de l'eau dans les modèles atmosphériques ;

- le développement et la généralisation de la technique d'assimilation des mesures de vapeur d'eau ;
- le développement de paramétrisations physiques plus adaptées ;

- la détermination et la quantification de l'amélioration de la prévision des précipitations résultant d'une meilleure caractérisation du champ de la vapeur d'eau.

\section{Remerciements}

Les auteurs remercient les personnes ayant contribué à la mise en œuvre du lidar Leandre 2 et des stations GPS pendant la campagne IHOP 2002 : Didier Bruneau et Pascal Genau du Service d'aéronomie ; Frédéric Blouzon, Patricia Delville, Abdel Abchiche et Nadir Amarouche de la Division technique de l'Insu ; Jean-Pierre Aubagnac du Centre national de recherches météorologiques et Philippe Collard du Laboratoire de géophysique, tectonique et sédimentologie. Les auteurs remercient également Jeff Besara (University of Oklahoma) ainsi que Jeff Keeler, Tim Rucker, Bruce Morley (National Center for Atmospheric Research) pour leur aide logistique pendant la campagne. Ces travaux ont été financés par le National Center for Atmospheric Research (Contrat $n^{\circ}$ S0230866) et le CNRS via les programmes nationaux Patom et PNTS.

\section{Bibliographie}

\footnotetext{
Bluestein H. B. et S. S. Parker, 1993 : Modes of isolated severe convective storm formation along the dryline. Mon. Wea. Rev., 121, $1354-1372$.

Bruneau D., P. Quaglia, C. Flamant, M. Meissonnier et J. Pelon, 2001 : The airborne lidar Leandre 2 for water vapor profiling in the troposphere. Appl. Opt., 40, 3450-3475.

Crook N. A., 1996 : Sensitivity of moist convection forced by boundary layer processes to low-level thermodynamic fields. Mon. Wea. Rev., 124, 1767-1785

Doerflinger E., 2001 : Les applications météorologiques du système de positionnement satellitaire GPS. La Météorologie 8érie, 34, 21-37.

- Ducrocq V., D. Ricard, J.-P. Lafore et F. Orain, 2002 : Storm-scale numerical rainfall prediction for five precipitating events over France: on the importance of the ini- tial humidity field. Wea. Forecasting, 17, 1236-1256.

Guichard F., D. Parsons et E. Miller, 2000 : Thermodynamical and radiative impact of the correction of sounding humidity bias in the tropics, J. Climate, 13, 3611-3624.

- Keeler R. J., T. Weckwerth, C. Flamant, F. Blouzon et J. Fox, 2002 : Leandre 2 horizontal water vapor Dial measurements. 21e International Laser Radar - Conference, Quebec, Canada, 8-12 juillet 2002.

- Lafore J.-P., J. Stein, N. Asencio, P. Bougeault, V. Ducrocq, J. Duron, C. Fischer, P. Hereil, P. Mascart, J.-P. Pinty, J.-L. Redelsperger, E. Richard et - J. Vila-Guerau de Arellano, 1998 : The Meso-NH atmospheric simulation system. Part I: adiabatic formulation and control simulation. Ann. Geophys., 16, 90-109.

- Uccellini L. W., P. J. Kocin et J. M. Sienkiewicz, 1994 : Advances in forecasting extratropical cyclogenesis at the National Meteorological Center. The Lifecycles of - Extratropical Cyclones, Proc. of An International Symp., vol. 1, ed. S. Gronas et M. A. Shapiro, 259-274

Weckwerth T. M., V. Wulfmeyer, R. M. Wakimoto, R. M. Hardesty, J. W. Wilson et R. M. Banta, 1999 : NCAR-NOAA Lower-Tropospheric Water Vapor Workshop. Bull. Amer. Meteor. Soc., 80, 2339-2358.

- Weckwerth T. M., 2000 : The effect of small-scale moisture variability on thunderstorm initiation. Mon. Wea. Rev., 128, 4017-4030.

Weckwerth T. M., D. B. Parsons, J. Moore, S. Koch, B. Demoz, P. Lemone, C. Flamant, B. Geerts, J. Wang et W. Feltz, 2003 : An overview of the International H2O Project (IHOP 2002) and some preliminary highlights, soumis à Bull. Amer. Meteor. Soc.

Xu K.-M., R. T. Cederwall, L. J. Donner, W. W. Grabowski, F. Guichard, D. E. Johnson, M. Khairoutdinov, S. K. Krueger, J. C. Petch, D. A. Randall, C. J. Seman, W.-K. Tao, J. J. Donghai Wang, J. J. Shao Cheng Xie, J. J. Yio et M.-H. Zhang, 2002 : An intercomparison of cloud-resolving models with the Atmospheric Radiation Measurement summer 1997 Intensive Observation Period data. Quart. J. Roy. Meteor. Soc., 128, 593-624.
} 